\title{
The Discovery of Host Galaxy Hi Absorption in CTA 21
}

\author{
C.J. Salter, ${ }^{1}$ D.J. Saikia, ${ }^{2,3}$ R. Minchin, ${ }^{1}$ T. Ghosh ${ }^{1}$ and Y. Chandola ${ }^{2}$ \\ 1 Arecibo Observatory, NAIC, HC3 Box 53995, Arecibo, Puerto Rico, PR 00612, USA \\ 2 National Centre for Radio Astrophysics, TIFR, Post Bag 3, Pune 411 007, India \\ ${ }^{3}$ ICRAR, University of Western Australia, Crawley, WA 6009, Australia
}

\begin{abstract}
We report the discovery of $\mathrm{HI}$ 21-cm absorption towards the well-studied $\mathrm{GHz}$ Peaked-Spectrum source CTA 21 (4C 16.09) using the Arecibo Telescope on 2009 September 20 and 21. Recently, the frequency band between 700 and $800 \mathrm{MHz}$ was temporarily opened up to radio astronomy when US TV stations were mandated to switch from analog to digital transmissions, with new frequency allocations. The redshifted Hi frequency for CTA 21 falls within this band. CTA 21 has a complex radio structure on a range of scales. The innermost prominent components are separated by $\sim 12$ mas while weak diffuse emission extends for up to $\sim 300$ mas. The Hi absorption profile that we find has two main components, one narrow, the other wider and blue-shifted. The total HI column density is $7.9 \times 10^{20} \mathrm{~cm}^{-2}$, assuming a covering factor of unity and a spin temperature of $100 \mathrm{~K}$. This Hi absorption confirms the recently determined optical redshift of this faint galaxy of $\mathrm{z} \sim 0.907$. We discuss this new detection in the light of $\mathrm{HI}$ absorption studies towards compact radio sources, and also the possibility that CTA 21 may be exhibiting multiple cycles of nuclear activity. This new detection in CTA 21 is consistent with a strong trend for detection of HI absorption in radio galaxies with evidence of episodic nuclear/jet activity.
\end{abstract}

Subject headings: galaxies: active — galaxies: nuclei — galaxies: individual (CTA 21) — radio lines: galaxies

\section{Introduction}

In the widely accepted model of active galactic nuclei (AGN), the source of energy is the gravitational potential energy of the material being accreted by a supermassive black hole via an accretion disk (e.g. Krolik 1999). This nuclear region is surrounded by a torus consisting of ionized, atomic and molecular gas components (e.g. Urry \& Padovani 1995 and references therein). Studying the kinematics and distribution of the gaseous components in the circumnuclear region is important for understanding a number of aspects, such as the fueling of the AGN activity, the anisotropy of the radiation field and thereby testing the unified schemes for active galaxies, interaction of the jets 
with the external gas clouds and probing star formation in the central regions of an AGN. At radio wavelengths, the ionized component may be investigated via polarization observations of compact radio structure in the nuclear regions of AGNs (e.g. Saikia \& Salter 1988; Mantovani et al. 1994; Udomprasert et al. 1997; Junor et al. 1999; Saikia \& Gupta 2003; Rossetti et al. 2009). These include the compact radio cores, the nuclear radio jets, and the compact steep spectrum (CSS) and Gigahertz peaked spectrum (GPS) sources. CSS sources are defined as having a projected linear size $<15 \mathrm{kpc}\left(\mathrm{H}_{o}=71 \mathrm{~km} \mathrm{~s}^{-1} \mathrm{Mpc}^{-1}, \Omega_{\mathrm{m}}=0.27\right.$, and $\left.\Omega_{\Lambda}=0.73\right)$, and a steep high-frequency radio spectrum $\left(\alpha>0.5\right.$, where $\left.\mathrm{S}(\nu) \propto \nu^{-\alpha}\right)$. GPS sources have spectra which turn over at, or above, $1 \mathrm{GHz}$, and are more compact than CSS objects whose spectra could turn over below $1 \mathrm{GHz}$. It is believed that GPS sources evolve into CSS objects, which later evolve to form the larger radio galaxies and quasars (Fanti et al. 1995; Readhead et al. 1996; O'Dea 1998; Snellen et al. 2000).

An important way of investigating atomic gas on subgalactic scales is via HI absorption towards the compact components of CSS and GPS sources or the radio nuclei of larger objects (e.g. van Gorkom et al. 1989; Conway \& Blanco 1995; Peck et al. 2000; Pihlström et al. 2003; Vermeulen et al. 2003; Gupta et al. 2006; Morganti et al. 2009 and references therein). These studies have shown that approximately $50 \%$ of GPS objects exhibit Hi absorption compared with about $35 \%$ for CSS sources (cf. Gupta et al. 2006). The Hi column density also exhibits an anticorrelation with source size (Pihlström et al. 2003; Vermeulen et al. 2003; Gupta et al. 2006). The Hi spectra exhibit a variety of line profiles with substantial red and blue shifts from the systemic velocities (Vermeulen et al. 2003; Gupta et al. 2006), suggesting that the atomic gas possesses complex motions, and may be out-flowing or in-falling, interacting with the jets, or rotating around the nucleus.

Another interesting class of AGN are those which exhibit signs of episodic activity. In radioloud AGN these can be seen as two or more pairs of radio lobes on opposite sides of the active nucleus, or as young radio lobes embedded in diffuse emission from earlier cycles of activity. The objects with two or more pairs of distinct lobes have been designated double-double radio galaxies or DDRGs (e.g. Schoenmakers et al. 2000; Saikia et al. 2006). Saikia et al. (2007) reported the detection of Hi absorption towards the central region of the DDRG J1247+6723, and suggested from available information that there might be a strong correlation between the detection of Hi in absorption and the occurrence of rejuvenated radio activity. The detection of Hi absorption in the rejuvenated radio galaxy 4C 29.30 is also consistent with this trend (Chandola et al. 2010).

In order to extend HI-absorption investigations to a larger number of GPS and CSS objects, especially those that are of lower luminosity or more distant, and also towards the cores of larger sources and the central regions of rejuvenated radio sources, we have been observing these sources both with the Arecibo 305-m telescope and the Giant Metrewave Radio Telescope (GMRT). The results obtained in the first phase of this study were reported by Gupta et al. (2006). Recently, the spectral region between 700 and $800 \mathrm{MHz}$ became temporarily available to radio astronomers due to the U.S. television switch from analog to digital transmissions, with new frequency allocations. We have used this opportunity to begin a search for highly redshifted $\mathrm{HI}$ and $\mathrm{OH}$ absorption within this band against the continuum emission from CSS/GPS radio sources of appropriate redshift. 
The first source observed, the well-known galaxy CTA 21 (4C 16.09), shows strong Hi absorption. We summarize the properties of CTA 21 in Section 2, the observations and results obtained with the Arecibo telescope in Section 3, and provide a discussion and concluding remarks in Section 4.

\section{CTA 21 (4C 16.09)}

This "classic" radio source has been studied extensively. A deep optical image of the field by Labiano et al. (2007) confirmed the earlier identification of the source by Stanghellini et al. (1993) with a faint galaxy having a $V$ magnitude of $\sim 23.4$. The optical spectrum shows a weak continuum with bright [OII] and [OIII] lines, and yields a redshift of 0.907 based on five lines (Labiano et al. 2007). The radio continuum spectrum of the source shows a turn-over at approximately $1 \mathrm{GHz}$, below which the spectrum is rather flat, or slightly inverted, down to at least $74 \mathrm{MHz}$ (Steppe et al. 1995; Torniainen et al. 2007). There is no evidence for significant radio variability of the source (Altschuler \& Wardle 1977; Bondi et al. 1996; Aller et al. 1985). It is at most very weakly polarized, this being $<0.5 \%$ at frequencies below $5 \mathrm{GHz}$, increasing to a few per cent at $15 \mathrm{GHz}$ (Perley 1982; Aller et al. 1985).

The high angular resolution radio structure of CTA 21 has been extensively imaged over the years. The early observations by Clarke et al. (1969) at 408 and $448 \mathrm{MHz}$, and Kellermann et al. (1971) at $1670 \mathrm{MHz}$ suggested the existence of structure on different scales although the details are difficult to relate to features seen in later images. Wilkinson et al. (1979) imaged the source at $609 \mathrm{MHz}$ and suggested that a compact component smaller than 12 mas contributes 4.6 Jy to the total flux density of $8.7 \mathrm{Jy}$, and is embedded in a $2.2-\mathrm{Jy}$ halo with a size of $30 \times 15 \mathrm{mas}^{2}$ at a position angle $(\mathrm{PA})$ of $160^{\circ}$. In addition, $1.9 \mathrm{Jy}$ is attributed to a fully resolved component which is $>30$ mas in size. Wilkinson et al. also consider the possibility that their "2.2-Jy halo" and their fully resolved component may be a single partially resolved component. Jones (1984) imaged the source at $4831 \mathrm{MHz}$ and found it to be an asymmetric double with a separation of 12 mas along a PA of $160^{\circ}$. He also noted that the components are slightly resolved perpendicular to the source axis. A combined MERLIN and VLBI image of CTA 21 at $1663 \mathrm{MHz}$ (Dallacasa et al. 1995) with an angular resolution of 40 mas shows a compact component which could correspond to the double structure seen by Jones (1984) with more diffuse extended emission to the south extending up to $\sim 300$ mas. Some evidence for this feature is seen in the $15-\mathrm{GHz}$ image by Spencer et al. (1989). The compact component, as imaged with a resolution of $\sim 4$ mas by Dallacasa et al., shows structure lying along the source axis, as well as emission orthogonal to this. On this basis, these authors suggest that it could be classified as a lobe. A VLBA image at $15 \mathrm{GHz}$ with $\sim 2.4$ mas resolution shows a number of components within a region of size $\sim 40$ mas (Kellermann et al. 1998). Comparing the images of Jones (1984) and Kellermann et al. (1998), which are closest in frequency and resolution, the southern, brighter component of Jones is possibly the brightest feature seen in the image of Kellermann et al., while the location and PA of the northern component of Jones would be consistent with the northern components seen by Kellermann et al. lying 10 mas from 
the dominant component. The weaker features of Kellermann et al. are not visible in the image of Jones (1984). Dallacasa et al. (1995) see emission extending farther south in their $1663-\mathrm{MHz}$ image when compared with the 15-GHz image of Kellermann et al. (1998).

\section{Observations and Results}

On 2009 June 12, and for a limited period, a largely unexplored region of frequency space between 700 and $800 \mathrm{MHz}$ opened up to radio astronomers when US TV stations were mandated to switch from analog to digital transmissions, with new frequency allocations. When aware of the opportunity to make observations in this temporarily freed-up band, the Arecibo Observatory prepared a receiver to cover it. This is used in conjunction with the Mock spectrometer (named after its designer/builder, the late Jeff Mock). Using this combination, we have undertaken a search of $29 \mathrm{CSS} / \mathrm{GPS}$ sources whose redshifts bring their $\lambda 21$-cm Hi or OH main lines into the 700-800 $\mathrm{MHz}$ band. At the time of the observations described here, a system temperature of $\sim 110 \mathrm{~K}$ was obtained on "cold sky". With a sensitivity of $\sim 9 \mathrm{~K} / \mathrm{Jy}$, this gave a System Equivalent Flux Density (SEFD) of $\sim 12.5 \mathrm{Jy}$.

The observations were carried out using the Double Position Switching (DPS) technique (Ghosh \& Salter 2002). Each ON/OFF position-switched observation with 5-min observing phases made on CTA 21, was followed by an ON/OFF of similar duration on the strong, angularly-nearby, continuum source, 3C79, which served as a band-pass calibrator. For sources with strong continuum emission, this strategy is needed to produce acceptable spectral baselines. In addition, apparent features seen in the spectra can often be recognized as astronomical, rather than due to radio frequency interference (RFI), from a comparison of the spectra for the target and calibrator source. Data reduction was performed using the Arecibo IDL analysis package written by Phil Perillat.

In initial observations for this project, we accumulated 35 min of "on-target" integration for the Hi line against CTA 21. The frequency of the band center was offset by $2 \mathrm{MHz}$ from that expected for the redshifted Hi line for CTA 21 since the Mock spectrometer is an AC-coupled device, producing a strong, but narrow, spike at the band center. The channel width of the spectra were $21 \mathrm{kHz}$, representing a velocity $(v=c z)$ resolution of $16.1 \mathrm{~km} \mathrm{~s}^{-1}$ at the frequency of the target line. Both orthogonal polarizations of the signal were recorded.

Individual ON/OFF scans on CTA 21 were processed to yield (ON-OFF) spectra, and these were bandpass corrected using corresponding spectra for 3C79. Each individual CTA 21 and 3C79 spectrum was inspected for quality, and any RFI present noted. All proved to be acceptable within the frequency range where the CTA $21 \mathrm{HI}$ absorption was expected to lie, and the bandpasscorrected spectra were then co-added and the orthogonal polarizations combined to produce a final spectrum. A strong HI absorption line was detected close to the expected redshift for CTA 21. A polynomial was fitted to the continuum spectrum of CTA 21, with the frequency range of the HI absorption masked out. This polynomial was then used to derive the fractional absorption across 
the line, as plotted against redshift in Figure 1. The fractional absorption has an rms noise of 0.00055 .

The Hi line that we detect has a fractional depth of $0.034 \pm 0.001$. It is broad, with a width to half power of $67 \mathrm{~km} \mathrm{~s}^{-1}$, and to zero intensity of $\approx 466 \mathrm{~km} \mathrm{~s}^{-1}$. The absorption spectrum, expressed as optical depth, $\tau$, was integrated to provide an estimate of the HI column depth, $N(\mathrm{HI})$, for the region of the host galaxy lying in front of the quasar continuum emission. This used;

$$
N(\mathrm{HI})=1.835 \times 10^{18} \frac{T_{\mathrm{s}} \int \tau(v) d v}{f_{c}} \mathrm{~cm}^{-2}
$$

where $T_{\mathrm{s}}$ is the Hi spin temperature (in $\mathrm{K}$ ), $f_{c}$ is the fraction of the background emission covered by the absorber, and $v$ is the radial velocity $(v=c z)$ in $\mathrm{km} \mathrm{s}^{-1}$. Assuming $T_{\mathrm{s}}=100 \mathrm{~K}$ and $f_{c}=1.0$, a value of $N(\mathrm{HI})=7.92 \times 10^{20} \mathrm{~cm}^{-2}$ results.

The CTA 21 absorption line was also fitted by multiple Gaussians to determine the peak optical depths $\left(\tau_{p}\right)$ and FWHMs $\left(\Delta v\right.$ in $\left.\mathrm{km} \mathrm{s}^{-1}\right)$ of likely individual components within the spectrum. The best fit was obtained using 4 Gaussian components, as displayed in Figure 2. The parameters derived from the fits are given in Table 1, and are discussed in Section 4. Here the Hi column density for each component has been determined using;

$$
N(\mathrm{HI})=1.93 \times 10^{18} \frac{T_{\mathrm{s}} \tau_{p} \Delta v}{f_{c}} \mathrm{~cm}^{-2}
$$

where $T_{\mathrm{s}}, f_{c}$ and $v$ are as above, and we again assume $T_{\mathrm{s}}=100 \mathrm{~K}$ and $f_{c}=1.0$.

\section{Discussion and Concluding Remarks}

With a total column density of $N(\mathrm{HI})=7.92 \times 10^{20} \mathrm{~cm}^{-2}$, CTA 21 is a source with a high HI column density. In the compilation of HI absorption observations towards CSS and GPS sources by Gupta et al. (2006), there are 96 sources in their 'full sample', of which only five (J0111+3906, $\mathrm{J} 1357+4354$, J1415+1320, J1819-6345, J1945+7055) have measured column densities which are higher. It is also the highest redshift CSS or GPS source for which HI absorption has been detected.

Table 1: Multiple-Gaussian fits to the Hi absorption spectrum of CTA 21.

\begin{tabular}{llclcc}
\hline $\begin{array}{l}\text { Id. } \\
\text { no. }\end{array}$ & $\begin{array}{l}\text { vhel } \\
\mathrm{km} \mathrm{s}^{-1}\end{array}$ & $\mathrm{z}$ & $\begin{array}{l}\text { FWHM } \\
\mathrm{km} \mathrm{s}^{-1}\end{array}$ & Frac. abs. & $\begin{array}{c}N(\mathrm{HI}) \\
10^{20} \mathrm{~cm}^{-2}\end{array}$ \\
\hline 1 & $271379(9.7)$ & 0.90522 & $31.8(20.0)$ & 0.0029 & 0.018 \\
2 & $271385(79)$ & 0.90524 & $212(77)$ & 0.0075 & 3.069 \\
3 & $271513(0.3)$ & 0.90567 & $47.6(0.8)$ & 0.0238 & 2.186 \\
4 & $271520(16)$ & 0.90569 & $131(32)$ & 0.0096 & 2.427 \\
\hline
\end{tabular}




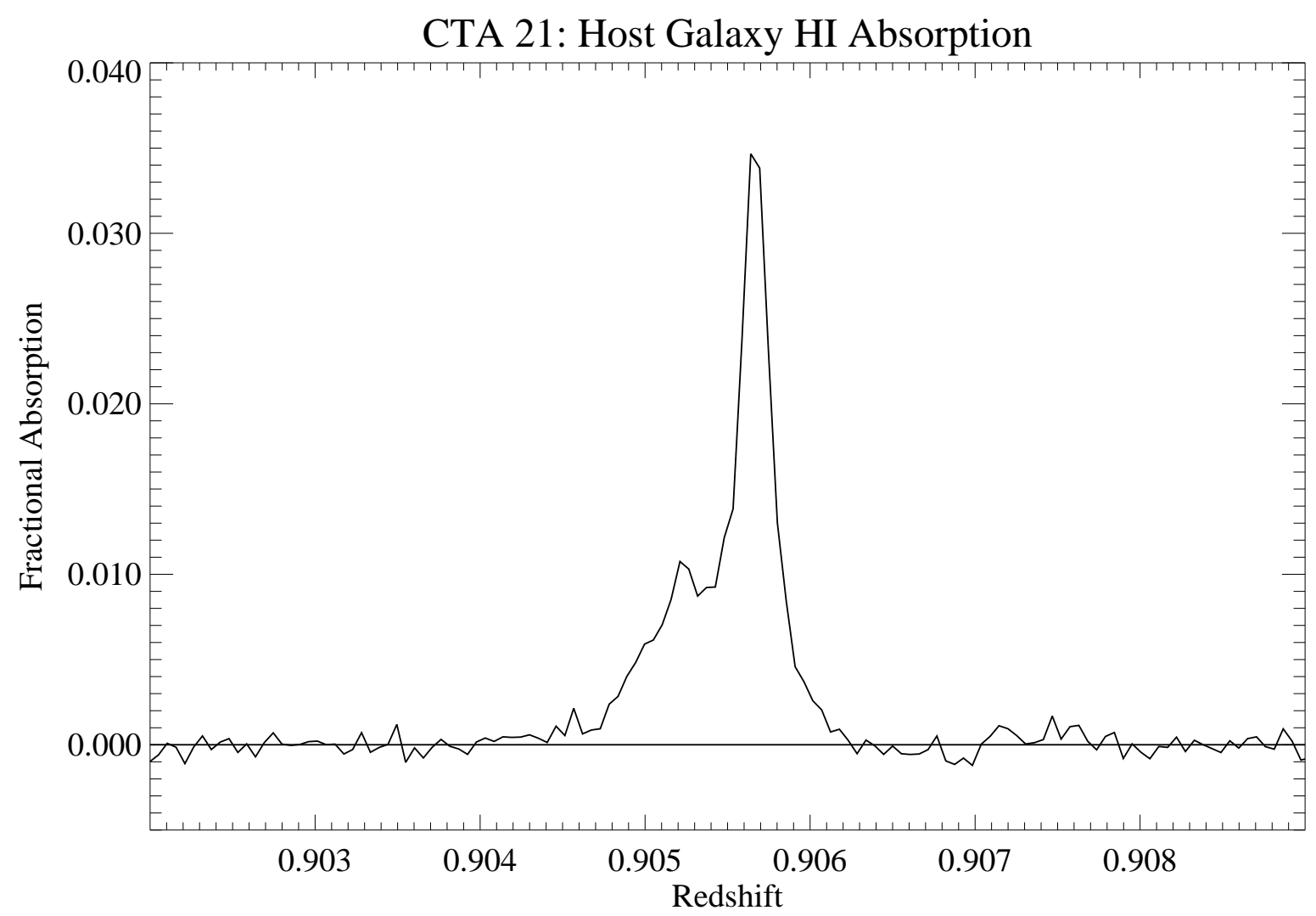

Fig. 1.- The Hi 21-cm fractional-absorption spectrum towards the GPS source CTA 21 plotted as a function of redshift. The fractional absorption has an rms noise of 0.00055 . 


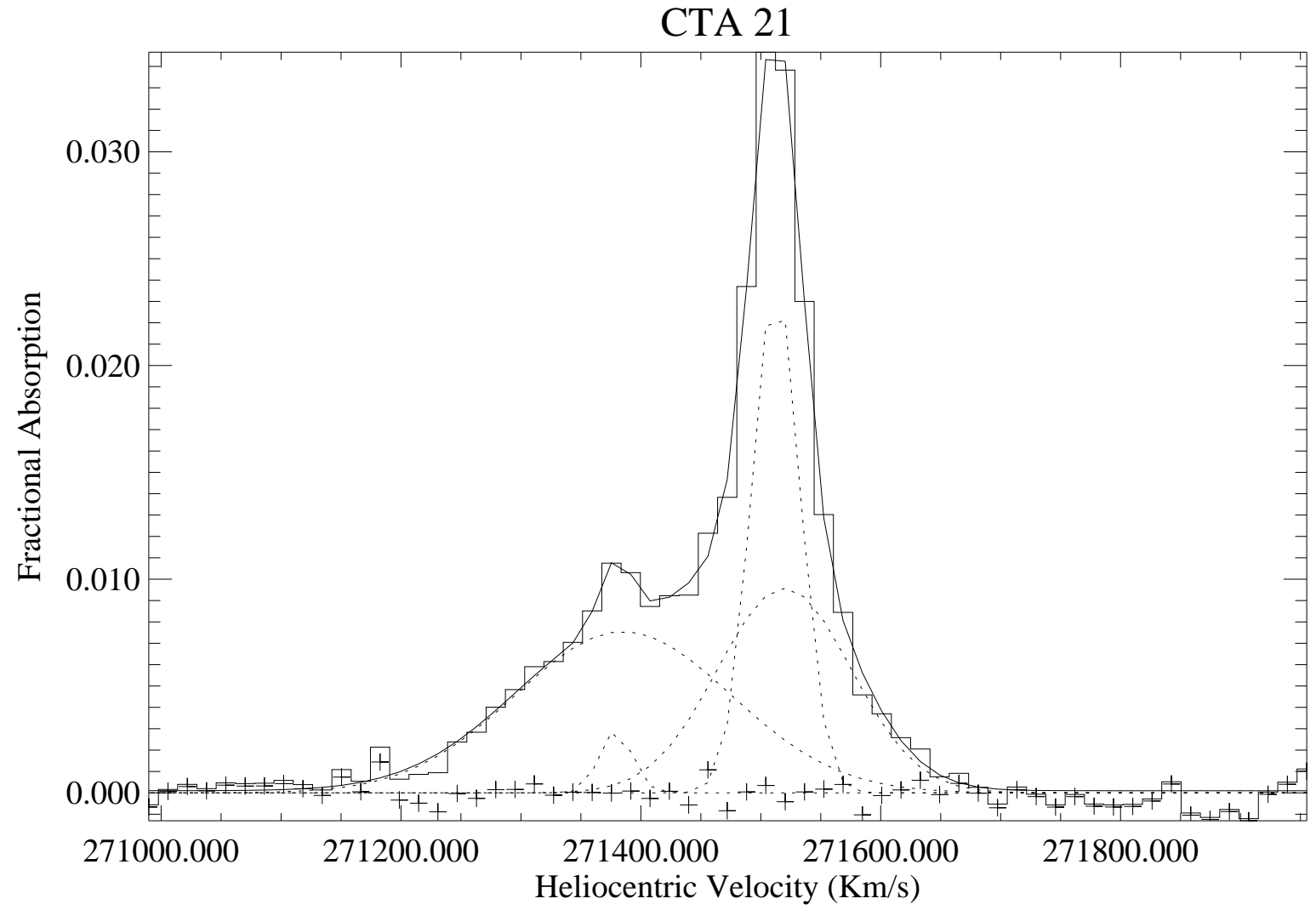

Fig. 2.- The best-fit Gaussian decomposition of the HI 21-cm fractional-absorption spectrum towards CTA 21 as a function of radial velocity. The four Gaussian components of the fit are plotted as dotted lines, while plus signs show the residual spectrum after subtraction of these components. 
With an overall linear size of $\sim 0.3 \mathrm{kpc}$, it is close to the upper envelope of the $N(\mathrm{HI})$ vs projected linear size diagram (Pihlström et al. 2003; Vermeulen et al. 2003; Gupta et al. 2006), and consistent with the overall trend. The redshift of the strongest absorption component in Table 1, $z \sim 0.9057$, agrees well with the optically-determined value of 0.907 (Labiano et al. 2007).

The highest-resolution VLBI images at $\sim 5$ and 15 GHz (Jones 1984; Kellermann et al. 1998), with angular resolutions of a few milliarcsec, do not show evidence for a core component. A comparison of these two images suggests that the prominent components have steep spectral indices. The estimated upper limit on the core flux density of $\sim 10 \mathrm{mJy}$ at $15 \mathrm{GHz}$ implies that the fraction of emission from the core is less than $\sim 1.5 \%$ at this frequency, which corresponds to an emitted frequency of $\sim 29 \mathrm{GHz}$. Although the $N(\mathrm{HI})$ vs core fraction diagram to test the unified scheme for active galaxies and probe the geometry of the Hi disk has a large scatter (e.g. Gupta \& Saikia 2006), CTA 21 appears broadly consistent with this. The non-detection of a radio core is also consistent with its identification as a radio galaxy.

The existence of a compact double-lobed structure of size $\sim 12$ mas seen in the highestresolution VLBI images (Jones 1984; Kellermann et al. 1998), plus the more extended diffuse emission discussed above, raises the possibility that CTA 21 may be undergoing repeated cycles of activity. For example, the image of Kellermann et al. (1998) shows evidence of weaker emission on opposite sides separated by $\sim 40$ mas, while Dallacasa et al. (1995) find evidence of diffuse emission extending up to $\sim 300$ mas towards the south. The different resolutions of these images make it difficult to reliably estimate spectral indices for the different components. However, on the basis of the compact double-lobed structure and diffuse extended emission we classify CTA 21 as a candidate rejuvenated radio galaxy. Rejuvenated radio sources cover a large range of linear sizes (see Saikia \& Jamrozy 2009, for a review), and it has been suggested that jet activity in compact radio sources may be intermittent on time scales of $\sim 10^{4}-10^{5}$ yr (Reynolds \& Begelman 1997). Saikia et al. (2007) and Chandola et al. (2010) explored a possible relationship between rejuvenation of radio or jet activity and the detection of $\mathrm{HI}$ in absorption. Unfortunately the number of sources is still small because most rejuvenated radio sources have weak radio emission in the central or nuclear region. The well-known examples of rejuvenated radio sources where Hi absorption has been detected are the giant radio galaxy $3 \mathrm{C} 236$ which has a projected linear size of $\sim 4250 \mathrm{kpc}$ and exhibits evidence of star formation (Conway \& Schilizzi 2000), the giant radio galaxy J1247+6723 with a GPS core (Saikia et al. 2007), the misaligned DDRG 3C293 (Beswick et al. 2004) which also exhibits fast outflowing gas blue-shifted by up to $\sim 1000 \mathrm{~km} \mathrm{~s}^{-1}$ (Emonts et al. 2005), the large southern radio galaxy Centaurus A (Sarma et al. 2002; Morganti et al. 2008), and the rejuvenated radio galaxy 4C 29.30 (Chandola et al. 2010). The archetypal radio galaxy Cygnus A, which has been shown to have two cycles of radio activity from radio and X-ray observations (Steenbrugge, Blundell \& Duffy 2008; Steenbrugge, Heywood \& Blundell 2010), also exhibits significant nuclear Hi absorption (Conway 1999). While the sample size needs to be increased, the detection of absorbing HI gas in rejuvenated galaxies appears to be even more frequent than for CSS and GPS objects. Considering the GPS objects listed by Gupta et al. (2006), these have the highest HI detection 
rate of $\sim 50 \%$, and a median column density of $\sim 3 \times 10^{20} \mathrm{~cm}^{-2}$. In comparison, the rejuvenated radio galaxies discussed here have column densities in the range of $\sim 8-50 \times 10^{20} \mathrm{~cm}^{-2}$, and tend to exhibit complex multi-component absorption profiles. The estimated column density of CTA 21 would be consistent with the range for the other rejuvenated radio galaxies.

\section{Acknowledgements}

We than an anonymous referee for his/her helpful comments. The Arecibo Observatory is part of the National Astronomy and Ionosphere Center, which is operated by Cornell University under a cooperative agreement with the National Science Foundation.

\section{REFERENCES}

Aller, H.D., Aller, M.F., Latimer, G.E., \& Hodge, P.E. 1985, ApJS, 59, 513

Altschuler, D.R., \& Wardle, J.F.C. 1977, MNRAS, 179, 153

Beswick, R.J., Peck, A.B., Taylor, G.B., \& Giovannini, G. 2004, MNRAS, 352, 49

Bondi, M., Padrielli, L., Fanti, R., Ficarra, A., Gregorini, L., \& Mantovani, F. 1996, A\&AS, 120, 89

Chandola, Y., Saikia, D.J., \& Gupta, N. 2010, MNRAS, 403, 269 (arXiv:0910.4427)

Clarke, R.W., Broten, N.W., Legg, T.H., Locke, J.L., \& Yen, J.L. 1969, MNRAS, 146, 381

Conway, J.E. 1999, ASPC, 156, 259

Conway, J.E., \& Blanco, P.R. 1995, ApJ, 449, 131

Conway, J.E., \& Schilizzi, R.T. 2000, in Conway J.E., Polatidis A.G., Booth R.S., Pihlström Y.M., eds, EVN Symp. 2000, Onsala Space Observatory, p. 123

Dallacasa, D., Fanti, C., Fanti, R., Schilizzi, R.T., \& Spencer, R.E. 1995, A\&A, 295, 27

Emonts B.H.C., Morganti R., Tadhunter C.N., Oosterloo T.A., Holt J., \& van der Hulst J.M. 2005, MNRAS, 362, 931

Fanti, C., Fanti, R., Dallacasa, D., Schilizzi, R.T., Spencer, R.E., \& Stanghellini, C. 1995, A\&A, 302,317

Ghosh, T., \& Salter, C.J. 2002, in ASP Conf. Ser. 278, Single-Dish Radio Astronomy: Techniques and Applications, eds. S. Stanimirovic, D. Altschuler, P. Goldsmith, \& C. Salter, (San Francisco: ASP), 521 
Gupta, N., \& Saikia, D.J. 2006, MNRAS, 370, 738

Gupta, N., Salter, C.J., Saikia, D.J., Ghosh, T., \& Jeyakumar, S. 2006, MNRAS, 373, 972

Jones, D. 1984, ApJ, 276, L5

Junor, W., Salter, C.J., Saikia, D.J., Mantovani, F., \& Peck, A.B. 1999, MNRAS, 308, 955

Kellermann, K.I., Vermeulen, R.C., Zensus, J.A., \& Cohen, M.H. 1998, AJ, 115, 1295

Kellermann, K.I., et al. 1971, ApJ, 169, 1

Krolik, J.H. 1999, Active galactic nuclei: from the central black hole to the galactic environment, Princeton University Press, Princeton

Labiano, A., Barthel, P.D., O’Dea, C.P., de Vries, W.H., Pérez, I., \& Baum, S.A. 2007, A\&A, 463, 97

Mantovani, F., Junor, W., Fanti, R., Padrielli, L., \& Saikia, D.J. 1994, A\&A, 292, 59

Morganti, R., Oosterloo, T., Struve, C., Saripalli, L. 2008, A\&A, 485, L5

Morganti, R., Peck, A.B., Oosterloo, T.A., van Moorsel, G., Capetti, A., Fanti, R., Parma, P., \& de Ruiter, H.R. 2009, A\&A, 505, 559

O’Dea, C.P. 1998, PASP, 110, 493

Peck, A.B., Taylor, G.B., Fassnacht, C.D., Readhead, A.C.S., \& Vermeulen R.C. 2000, ApJ, 534, 104

Perley, R.A. 1982, AJ, 87, 859

Pihlström, Y.M., Conway, J.E., \& Vermeulen R.C. 2003, A\&A, 404, 871

Readhead, A.C.S., Taylor, G.B., Pearson, T.J., \& Wilkinson, P.N. 1996, ApJ, 460, 634

Reynolds, C.S., \& Begelman, M.C. 1997, ApJ, 487, L135

Rossetti, A., Mantovani, F., Dallacasa, D., Junor, W., Salter, C.J., \& Saikia, D.J. 2009, A\&A, 504, 741

Saikia, D.J., \& Gupta, N. 2003, A\&A, 405, 499

Saikia, D.J., \& Jamrozy, M. 2009, BASI, 37, 63 (arXiv:1002.1841)

Saikia, D.J., \& Salter, C.J. 1988, ARA\&A, 26, 93

Saikia, D.J.; Gupta, N., \& Konar, C. 2007, MNRAS, 375, L31

Saikia, D.J., Konar, C., \& Kulkarni, V.K. 2006, MNRAS, 366, 1391 
Sarma A.P., Troland T.H., \& Rupen M.P. 2002, ApJ, 564, 696

Schoenmakers, A.P., de Bruyn, A.G., Roettgering, H.J.A., van der Laan, H., \& Kaiser, C.R. 2000, MNRAS, 315, 371

Snellen, I.A.G., Schilizzi, R.T., Miley, G.K., de Bruyn, A.G., Bremer, M.N., \& Röttgering, H.J.A. 2000, MNRAS, 319, 445

Spencer, R.E., McDowell, J.C., Charlesworth, M., Fanti, C., Parma, P., \& Peacock, J.A. 1989, MNRAS, 240, 657

Stanghellini, C., O’Dea, C.P., Baum, S.A., \& Laurikainen E. 1993, ApJS, 88, 1

Steenbrugge K.C., Blundell K.M., \& Duffy P. 2008, MNRAS, 388, 1465

Steenbrugge K.C., Heywood I., \& Blundell K.M. 2010, 401, 67

Steppe, H., Jeyakumar, S., Saikia, D.J., \& Salter, C.J. 1995, A\&AS, 113, 409

Torniainen, I., Tornikoski, M., Lähteenmäki, A., Aller, M.F., Aller, H.D., \& Mingaliev, M.G. 2007, A\&A, 469, 451

Udomprasert, P.S., Taylor, G.B., Pearson, T.J., \& Roberts, D.H. 1997, ApJ, 483, L9

Urry, M.C., \& Padovani, P. 1995, PASP, 107, 803

van Gorkom, J.H., Knapp, G.R., Ekers, R.D., Ekers, D.D., Laing, R.A., \& Polk, K.S. 1989, AJ, 97, 708

Vermeulen R.C., et al. 2003, A\&A, 404, 861

Wilkinson, P.N., Readhead, A.C.S., Anderson, B., \& Purcell, G.H. 1979, ApJ, 232, 365

This preprint was prepared with the AAS LATEX macros v5.2. 